\title{
Relation between Renal Resistive Index and Serum Albumin in Nephrotic Syndrome
}

\author{
Pranoy Dey ${ }^{1}$, Niku Hage ${ }^{2}$ \\ ${ }^{1,2}$ Department of Paediatrics, Assam Medical College and Hospital, Dibrugarh, Assam, India.
}

\section{ABSTRACT}

\section{BACKGROUND}

Renal resistive index (RRI) is a non-invasive marker of renal function, which can be measured by renal USG Doppler. High RI indicates poor prognosis, which has been associated with conditions such as proteinuria, hypertension, chronic kidney disease and renal failure. As nephrotic syndrome is characterised by significant proteinuria and hypoalbuminemia, which may progress to renal failure, it is important to see the correlation of RRI with serum albumin level. The purpose of this study was to assess the relation between renal resistive index and serum albumin in nephrotic syndrome.

\section{METHODS}

USG renal Doppler was done for all the children with nephrotic syndrome and RRI was calculated as [(peak - systolic velocity - end - diastolic velocity) / peak - systolic velocity]. Blood investigations were also done, from which serum albumin values were obtained. RRI was compared with mean serum albumin level. Statistical analysis of data was performed using the computer programme, Statistical Package for Social Sciences (SPSS for windows, version 21.01, Chicago, SPSS Inc.) and Microsoft excel 2010. Results on continuous measurement were presented as mean \pm standard deviation and compared using Analysis of Variance (ANOVA).

\section{RESULTS}

The mean serum albumin in the children with nephrotic syndrome in our study was $1.82 \pm 0.32 \mathrm{gm} / \mathrm{dl}$. The mean RI value was $0.66 \pm 0.14 \mathrm{SD}, 0.64 \pm 0.15 \mathrm{SD}$ and $0.66 \pm$ $0.12 \mathrm{SD}$ as well as $0.63 \pm 0.12 \mathrm{SD}, 0.67 \pm 0.16 \mathrm{SD}$ and $0.71 \pm 0.13 \mathrm{SD}$ on the right and left kidney respectively for each of upper, middle, and lower pole. $\mathrm{P}$ value of left kidney and right kidney was 0.0057 and 0.6409 respectively.

\section{CONCLUSIONS}

Lower the mean serum albumin level, higher the range of RRI.

\section{KEY WORDS}

Serum Albumin, Renal Resistive Index, Nephrotic Syndrome, USG Renal Doppler
Corresponding Author:

Dr. Pranoy Dey,

Department of Paediatrics,

Assam Medical College and

Hospital, Dibrugarh,

Assam, India.

E-mail: pranoydey241@gmail.com

DOI: $10.14260 / \mathrm{jemds} / 2021 / 456$

How to Cite This Article:

Dey $P$, Hage N. Relation between renal resistive index and serum albumin in nephrotic syndrome. J Evolution Med Dent Sci 2021;10(30):2228-2232, DOI: $10.14260 / \mathrm{jemds} / 2021 / 456$

Submission 06-12-2020,

Peer Review 21-05-2021,

Acceptance 25-05-2021,

Published 26-07-2021.

Copyright (c) 2021 Pranoy Dey et al. This is an open access article distributed under Creative Commons Attribution License [Attribution 4.0 International (CC BY 4.0)] 


\section{BACKGROUND}

Renal resistive index (RI) is a non-invasive marker of renal function that is derived from the calculation of the relationship between systolic and diastolic renal arterial pressure. It is an indicator of the resistance to flow within the kidneys. RI has been shown as a prognostic instrument in assessing the progression of renal disease, especially in hypertension and proteinuria. ${ }^{1}$ An initial high RI denotes poor prognosis. This eventually leads to more disease progression. RI is commonly used as an index of intrarenal arterial resistance. Studies have demonstrated that high RI, proteinuria, and hypertension are known risk factors for the progression of chronic renal disease though an association between RI and specific histological subtype has not been shown to be of statistical significance. ${ }^{2}$ Normal RI is approximately 0.6 (ranging between 0.56 and 0.66 ). The generally acceptable normal value of the renal RI is taken as $\leq 0.7 .3$

However, a slightly higher RI value $(0.72 \pm 0.03)$ has been shown in healthy young children of age four and a half years and below. Thus, the RI value of 0.70 is only applicable for older children. ${ }^{4}$ A significant correlation has been shown between RI, glomerulosclerosis and focal interstitial fibrosis. ${ }^{3}$ In general, higher levels of RI are associated with worse renal parenchymal damage and worse outcome. However, whether increased RI affects responsiveness to steroids remains unknown. Doppler ultrasonography is a non-invasive method widely used in clinical practice for chronic kidney disease (CKD) patients, which is used for determining the renal resistive index. It can detect not only renal macro abnormalities, but also changes in the renal vasculature.

RI is determined from USG Doppler by assessing the systolic and diastolic blood velocity in segmental arteries and applying the following formula (peak systolic velocity- end diastolic velocity/ peak systolic velocity). Evaluation of vascular impedance at different sites of renal parenchyma may suggest functional or structural changes within the kidneys. The relationship between renal histological changes and RI has been investigated previously, however results were not always consistent. Moreover, vascular complications in patients with nephrotic syndrome are an uncommon but serious problem.

Nephrotic syndrome is characterized by heavy range proteinuria ( $>40 \mathrm{mg} / \mathrm{m}^{2} / \mathrm{hr}$ or $>3.5 \mathrm{~g} / 24 \mathrm{hr}$ ), resulting in significant decrease in serum albumin level (hypoalbuminemia $\leq 2.5 \mathrm{~g} / \mathrm{dl}$ ) which are important diagnostic criterion that define nephrotic syndrome.

It is a major cause of chronic renal disease among paediatric age group. 80 - $90 \%$ of children with idiopathic nephrotic syndrome respond to daily glucocorticoid therapy, however recent data suggests that over $30 \%$ of steroid sensitive nephrotic syndrome (SSNS) children relapse in adulthood. ${ }^{4,5}$

It may be complicated by renal failure, especially in cases which are steroid resistant or steroid dependent. Therefore, this study was done to assess the relation between serum albumin (which is very low in nephrotic syndrome) and RRI which might help in prognostication of renal damage or early identification of progressive renal dysfunction.

\section{METHODS}

In a hospital based analytical study, all the children (63 children) with nephrotic syndrome attending tertiary care centre (Assam Medical College and Hospital) and admitted in our ward were taken for the study, which was conducted from May 2019 to June 2020. This included children up to 12 yrs. after a proper informed consent was taken from the guardian. Ethical clearance was taken from the Institutional Ethics Committee. Detailed history, clinical examination and relevant laboratory and radiological investigations, including colour Doppler ultrasonographic study of renal artery was done.

The received data along with the details regarding age, gender, locality, socioeconomic status, clinical features was recorded in a predesigned proforma. The serum albumin and creatinine were derived by analysing the subjects' serum, and were compared to the local standardized laboratory values. The urinalysis was however derived by using the qualitative method of dipstick, using the standardized colour code. Nephrotic syndrome was characterized by significant (Nephrotic Range) proteinuria $>40 \mathrm{mg} / \mathrm{m}^{2} / \mathrm{hr}$, hypoalbuminemia $\leq 2.5 \mathrm{~g} / \mathrm{dl}$, oedema, and hyperlipidaemia (cholesterol $>200 \mathrm{mg} / \mathrm{dl}$ ).

The study was performed by Toshiba Aplio 500 using 2.5 to 5 - MHz curved array transducers for adequate depth of penetration to visualize the abdominal aorta and its major branches: celiac, mesenteric, and renal arteries. The first step was to optimize the gray-scale and colour Doppler parameters so as to improve renal artery visualization as well as the conspicuity of flow reducing lesions. Adjustment of the colour Doppler parameters, including colour gain, PRF (colour velocity scale), and wall filter, was performed in areas of laminar flow, in either the aorta or a normal segment of a renal artery. Proper colour Doppler adjustment was done to "screen" the vessel quickly for stenosis, because elevated velocities in stenotic regions then produce a colour aliasing artifact that is readily apparent. Our sonographer preferred the decubitus or oblique positions because they can use the liver and kidneys as acoustic windows to visualize the renal arteries. The spectral Doppler examination was performed with a small sample volume so as to obtain flow information from only the vessel of interest. Pulsed Doppler sampling was performed with angles of 60 degrees or less. Angles of greater than 60 degrees were never used, because this artifactually increases the PSV measurement. The PRF was adjusted so that the waveforms are large and easy to read but without causing aliasing. Left and right decubitus patient positions were preferred for the kidney examination (left decubitus for the right kidney and vice versa). Echogenicity and thickness of the renal parenchyma were noted to measure the kidney length. The kidneys were assessed for atrophy, scarring, hydronephrosis, calculi, or masses. A longitudinal survey of the abdominal aorta was performed from the celiac artery to the iliac bifurcation and evaluate the amount of atherosclerotic plaque. This was done with both gray-scale and colour flow Doppler.

Gray-scale evaluation was important to assess for irregular plaque and ostial lesions (i.e., at the origin of the aortic branches), which may be obscured by colour flow blooming. Flow abnormalities at the origin of the celiac and superior mesenteric arteries were looked for that indicates significant stenosis. The size and location of abdominal aortic aneurysms 
were noted. Finally, angle - corrected PSV measurements were obtained from the abdominal aorta at the level of the renal arteries. These aortic velocity measurements were used to determine the renal artery-aorta velocity ratio.

The right renal artery was often easier to identify than the left with this approach and was relatively easy to follow to the renal hilum. The left renal artery was harder to follow all the way to the kidney from an anterior approach. The left renal artery was better seen by positioning the patient in a right lateral decubitus position and scanning from a left posterolateral transducer approach, using the left kidney as an acoustic window. Transverse and sagittal sweeps of the abdominal aorta and kidneys were performed to identify duplicate renal arteries. These arteries may arise from the inferior aorta or iliac arteries and were followed to the renal hilum or either pole of the kidney. Each renal artery was examined with colour flow imaging from its origin to the hilum of the kidney, including the main hilar branches. We looked for areas of high-velocity flow, indicated by colour shifts or aliasing, as well as turbulence related flow disturbances, as these may be related to stenosis. We routinely had obtained PSV measurements from the origin, proximal, mid, and distal segments of each renal artery. A small sample volume (1.5 - 2.0 $\mathrm{mm}$ ), and an angle of insonation of 60 degrees or less were used. Finally, waveforms were also obtained from the segmental arteries in the upper, mid, and lower poles of each kidney. Thus, at least seven waveforms were captured from each side. It was important to obtain clean, crisp waveforms with well-defined borders for analysis. This was accomplished by adjusting the spectral display so that the waveforms were large and easily measured. This allowed the examiner to readily determine the PSV, acceleration time or index, and the resistive index (RI). The RI is the PSV minus the end - diastolic velocity, divided by the PSV. The generally acceptable normal value of the renal RI is taken as $\leq 0.7{ }^{3}$. However, a slightly higher RI value $(0.72 \pm 0.03)$ has been shown in healthy young children of age four and a half years and below.

\section{Statistical Analysis}

The statistical analysis of data was performed using the computer programme, Statistical Package for Social Sciences (SPSS for windows, version 21.01, Chicago, SPSS Inc.) and Microsoft excel 2010. Results on continuous measurement presented as mean \pm standard deviation were compared using Analysis of Variance (ANOVA). When the P -value was found significant $(\mathrm{P}<0.05)$ among 3 groups, post hoc analysis was done to find out the significance between 2 individual groups. Discrete data were expressed as numbers (\%) and were analysed using chi-square and Fischer's exact test (where the cell counts were $<5$ or 0 ). Pearson's correlation coefficient $\AA$ was used to measure the associations among continuous variables. For all analysis, the statistical significance was fixed at $5 \%$ level. ( $\mathrm{P}$ value $<0.05)$.

\section{RESULTS}

The mean age of the children included in the study was $6.39 \pm$ 2.62 years. The sex distribution of the cases is tabulated in Table 5.2 and is represented in Fig 5.2. Out of 63 nephrotic patients included, $60.32 \%$ (38) were males and $39.68 \%$ (25) were females with a male: female ratio of $1.52: 1$. Idiopathic nephrotic syndrome was more prevalent in boys than in girls $(2: 1)$
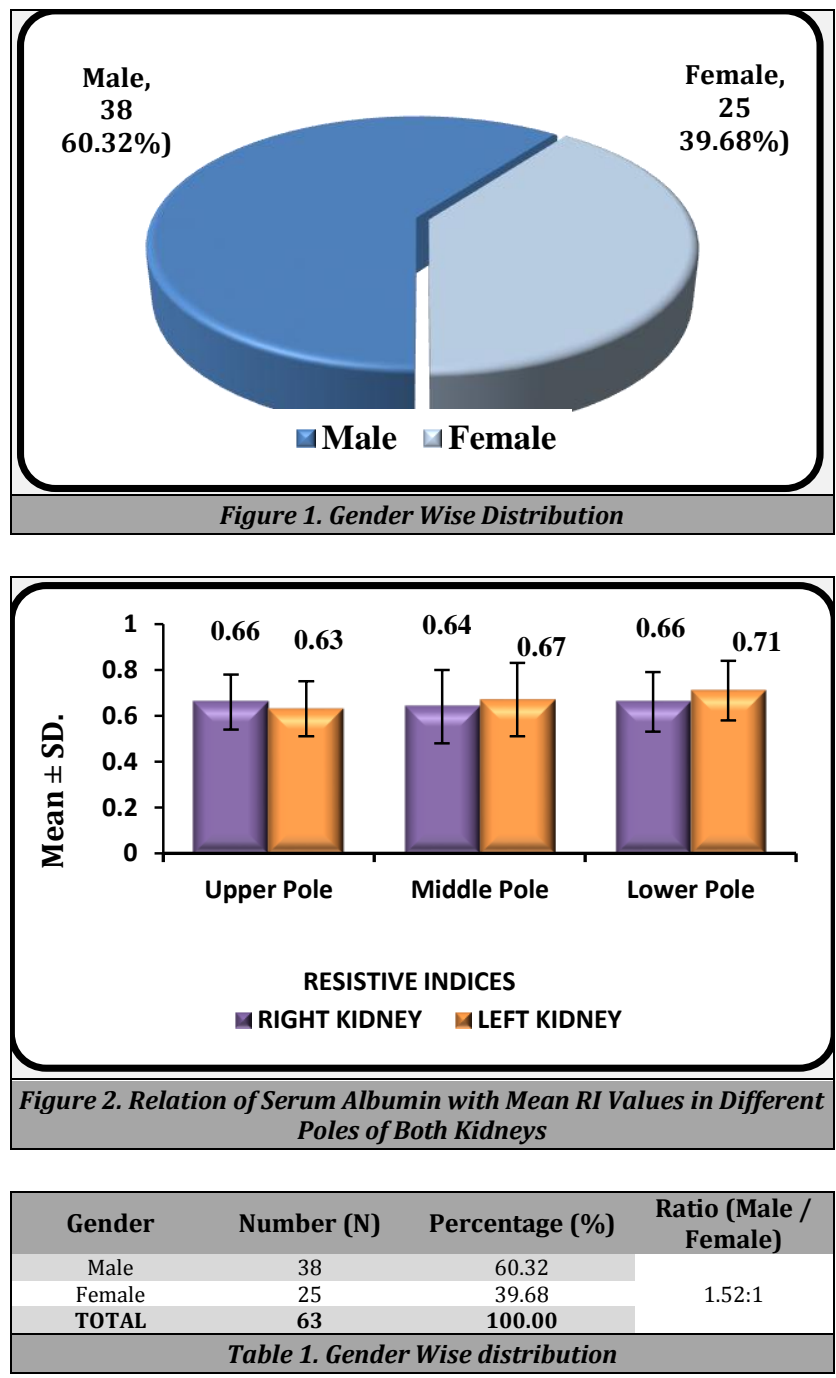

\begin{tabular}{|cc|}
\hline & Mean \pm SD \\
Serum Albumin $(\mathrm{gm} / \mathrm{dl})$ & $1.82 \pm 0.32 \mathrm{gm} / \mathrm{dl}$ \\
\hline Table 2. Serum Albumin \\
\hline
\end{tabular}

\begin{tabular}{|c|c|c|c|c|c|c|c|c|}
\hline \multirow{2}{*}{$\begin{array}{c}\text { Serum } \\
\text { Albumin } \\
\text { (gm / dl) }\end{array}$} & \multicolumn{3}{|c|}{ Right Kidney } & \multicolumn{4}{|c|}{ Left Kidney } & \multirow[b]{2}{*}{ P Value } \\
\hline & $\mathbf{N}$ & Mean & SD & P Value & $\mathbf{N}$ & Mean & SD & \\
\hline$\leq 2.5 \mathrm{gm} / \mathrm{dl}:$ & & & & & & & & \\
\hline Upper Pole & 63 & 0.66 & 0.14 & \multirow{3}{*}{0.6409} & 63 & 0.63 & 0.12 & \multirow{3}{*}{0.0057} \\
\hline Middle Pole & 63 & 0.64 & 0.15 & & 63 & 0.67 & 0.16 & \\
\hline Lower Pole & 63 & 0.66 & 0.12 & & 63 & 0.71 & 0.13 & \\
\hline
\end{tabular}

In all the cases included in the study, serum albumin was less than $2.5 \mathrm{gm} / \mathrm{dl}$ and mean value was found out to be 1.82 $\pm 0.32 \mathrm{gm} / \mathrm{dl}$. The relation between serum albumin and mean RRI values in different poles of both kidneys was seen (Table 2 , Fig 2). All the cases presented with the serum albumin value of less than $2.5 \mathrm{gm} / \mathrm{dl}$. The mean RI value was $0.66 \pm 0.14 \mathrm{SD}$, $0.64 \pm 0.15 \mathrm{SD}$ and $0.66 \pm 0.12 \mathrm{SD}$ as well as $0.63 \pm 0.12 \mathrm{SD}$, $0.67 \pm 0.16 \mathrm{SD}$ and $0.71 \pm 0.13 \mathrm{SD}$ on the right and left kidneys respectively for each of the upper, middle, and lower pole. The relation between the lower serum albumin value and RI of left kidney was found out to be statistically significant with a $\mathrm{P}$ 
value of 0.0057 . But this was statistically insignificant in case of right kidney (P value 0.6409).

\section{DISCUSSION}

Out of 63 nephrotic patients included in the study, preschool children $\leq 5$ years were $23.81 \%$ (15) and school going age group > 5 years was $76.19 \%$ (48). The mean age of the children included in the study was $6.39 \pm 2.62$ years. In study done by A. Safaei et al. ${ }^{6}$ the mean age of the children was found to be $4.87 \pm 3.24$ years. In the study conducted by el Bakkali $\mathrm{L}$ et al. ${ }^{7}$ the mean age of children was 5.08 years. But this study considered age group up to 18 years. In other studies, like, the ones done by McKinney PA et al. ${ }^{8}$ also showed similar kind of results with a mean age of 4.5 years.

Our study showed male:female ratio of 1.52:1. Hence showing male predominance which is similar to other studies. The preponderance of nephrotic syndrome in boys is well established and reportedly confined to patients with minimal lesions. Srivastava et al. and Solani et al. studies demonstrated the male:female ratio of 2:1.9 Gulati et al. also showed male preponderance in the study. ${ }^{10}$ In the study conducted by A. Safaei et al. they also found a male predominance with 29 boys (66\%) and 15 girls (34\%) and with a male:female ratio of 1.9 $/ 1$.

Majority of the children $<5$ years had normal nutritional status with appropriate growth. Some overweight cases found in the study were due to the oedematous status of the child resulting in false increase in weight. Syndrome $\geq 5 \mathrm{yrs}$, weight / age and height / age were within the normal limits for all the children. However, as per BMI, it showed that $27.08 \%(13$ children) were overweight with $\mathrm{BMI}>23^{\text {rd }}$ adult equivalent and $4.17 \%$ (2) were obese with BMI $>27^{\text {th }}$ adult equivalent, which could be attributed to the oedematous condition of the child. While $4.17 \%$ (2) were severely wasted with BMI $<3^{\text {rd }}$ percentile. From the nutritional status of children with nephrotic syndrome analysed in our study we found that out of 63 nephrotic patients, $95.24 \%$ (60) had normal nutritional status, $1.59 \%$ (1) had moderate malnutrition and $3.17 \%$ (2) had severe malnutrition. No patients showed mild malnutrition. Thus correlating well with the socioeconomic status of the family, where majority of them in this study belonged to the upper lower class family $38.10 \%$ (24).

In our study, as only the nephrotic syndrome cases were taken, all the cases had shown the serum albumin level of less than $2.5 \mathrm{gm} / \mathrm{dl}$ by definition. The mean value of serum albumin was found out to be $1.82 \pm 0.32 \mathrm{gm} / \mathrm{dl}$. In the study conducted by Hossain M. et al.11 the mean value of serum albumin was found out to be $1.88 \pm .37 \mathrm{gm} / \mathrm{dl}$ which is similar to our study finding. Study by Sapartini G. et al.12 the mean value of serum albumin was found to be $1.4 \pm 0.4 \mathrm{gm} / \mathrm{dl}$. In the study by Roy R. et al. ${ }^{13}$ the mean value of serum albumin was $1.60 \pm 0.25 \mathrm{gm} / \mathrm{dl}$ which was lower than our study findings. In the study by Sah J et al. it was found that mean serum albumin was $2.15 \pm 0.07 \mathrm{gm} / \mathrm{dl}$, which was higher compared to the present study. ${ }^{14}$

The relation between the serum albumin levels and the RI value of different poles of kidney was also seen in this study. The mean RI value was ranging from $0.64 \pm 0.15 \mathrm{SD}$ and $0.66 \pm$ $0.14 \mathrm{SD}$ and $0.63 \pm 0.12 \mathrm{SD}-0.71 \pm 0.13 \mathrm{SD}$ on the right and left kidney respectively. The relation between the decreasing serum albumin value and RI of left kidney was found out to be statistically significant with a p value of 0.0057 . But this was statistically insignificant in case of right kidney ( $P$ value 0.6409). But study conducted by Omolola M. A. et al. ${ }^{15}$ had found no significant correlation between the serum albumin level and RI of kidneys. There were also not many studies done to see this relationship between serum albumin levels and RI value of different kidneys.

From our study, we have seen that RRI in left kidney was in higher range for mean serum albumin $1.82 \pm 0.32 \mathrm{gm} / \mathrm{dl}$. Therefore, relationship between RRI and serum albumin may give indirect information on worsening renal function and thus renal prognosis.

\section{CONCLUSIONS}

The resistive index is a known non-invasive tool in assessing the renal function in kidney diseases, especially in proteinuria conditions like Paediatric nephrotic syndrome (NS) which is a glomerular disease. However, there is a limited knowledge in the use of Doppler Resistive in the management and prognostication of this disease condition and very few studies on the same.

As per our study, we have found that there is a significant correlation between serum albumin and RI of left kidney due to lack of evidence and limited studies, USG renal Doppler cannot be used for prognostication of the disease severity in cases with nephrotic syndrome and also to predict the disease outcome. However, it can be useful to indirectly assess the renal function by comparing with serum albumin.

Data sharing statement provided by the authors is available with the full text of this article at jemds.com.

Financial or other competing interests: None.

Disclosure forms provided by the authors are available with the full text of this article at jemds.com.

\section{REFERENCES}

[1] Parolini C, Noce A, Staffolani E, et al. Renal resistive index and long-term outcome in chronic nephropathies. Radiology 2009;252(3):888-96.

[2] Sugiura T, Wada A. Resistive index predicts renal prognosis in chronic kidney disease. Nephrol Dial Transplant 2009;24(9):2780-5.

[3] Mostbek GH, Kain R, Mallek R, et al. Duplex doppler sonography in renal parenchymal disease: histopathologic correlation. J Ultrasound Med 1991;10(4):189-94.

[4] Ruth EM, Kemper MJ, Leumann EP, et al. Children with steroid-sensitive nephrotic syndrome come of age: longterm outcome. J Pediatr 2005;147(2):202-7.

[5] Fakhouri F, Bocquet N, Taupin P, et al. Steroid-sensitive nephrotic syndrome: from childhood to adulthood. Am J Kidney Dis 2003;41(3):550-7.

[6] Safaei A, Maleknejad S. Spectrum of childhood nephrotic syndrome in Iran: a single center study. Indian J Nephrol 2009;19(3):87-90. 
[7] El Bakkali L, Pereira RR, Kuik D, et al. Nephrotic syndrome in the Netherlands: a population-based cohort study and a review of the literature. Pediatr Nephrol 2011;26(8):1241-6.

[8] McKinney PA, Feltbower RG, Brocklebank JT, et al. Time trends and ethnic patterns of childhood nephrotic syndrome in Yorkshire, UK. Pediatr Nephrol 2001;16(12):1040-4.

[9] Srivastava T, Simon SD, Alon US. High incidence of focal segmental glomerulosclerosis in nephrotic syndrome of childhood. Pediatr Nephrol 1999;13(1):13-8.

[10] Gulati S, Sural S, Sharma RK, et al. Spectrum of adolescentonset nephrotic syndrome in Indian children. Pediatr Nephrol 2001;16(12):1045-8.

[11] Hossain M, Deb KP, Hossain MA, et al. Correlation between serum cholesterol and serum albumin level in childhood nephrotic syndrome. Urol Nephrol Open Access J 2016;3(4):86-90.
[12] Sapartini G, Rachmadi D, Garna H. Correlation between serum albumin and creatinine levels in children with nephrotic syndrome. Paediatrica Indonesiana 2016;48(6):354.

[13] Roy R, Islam M, Jesmin T, et al. Prognostic value of biochemical and hematological parameters in children with nephrotic syndrome. Journal of Shaheed Suhrawardy Medical College 2013;5(2):95-8.

[14] Sah JP, Pandey R, Jaiswal S, et al. Correlation of hypoproteinemia and hypoalbuminemia with hypercholesterolemia in the children with nephrotic syndrome. Research and Reviews: A Journal of Health Professions 2013;3(2):1-11.

[15] Atalabi OM, Afolabi OS, Asinobi AO. Renal doppler indices in children with nephrotic syndrome: findings from a tertiary hospital in Nigeria. Niger J Physiol Sci 2020;30(12):17-23. 